\title{
The hidden value of air transportation infrastructure
}

\author{
Bruno Miller \\ International Center for Air Transportation, Massachusetts Institute of Technology \\ Rm. 35-217, 77 Massachusetts Ave, Cambridge, MA 02139. Tel: (617) 2537748 \\ brunom $@$ mit.edu \\ John-Paul Clarke \\ International Center for Air Transportation, Massachusetts Institute of Technology \\ Rm. 33-408, 77 Massachusetts Ave, Cambridge, MA 02139. Tel: (617) 2530904 \\ johnpaul@mit.edu
}

\section{Abstract}

Air transportation is a key strategic asset in that it provides access to markets and thereby enables the economic development of nations. Thus, in order to maintain their competitiveness in a global economy, countries must invest in air transportation infrastructure to ensure their ability to meet current and future demand for aviation services. The objective of this paper is to develop and illustrate a methodology for evaluating the strategic value of air transportation infrastructure, in particular the benefits associated with the ability to react quickly to changes in the market. The hypothesis is that by recognizing and taking advantage of this strategic value, it may be possible to design better policies for aviation infrastructure delivery.

The methodology developed here uses system dynamics to model different strategies for infrastructure delivery. These strategies are defined by three variables: the amount of capacity increase, the time to deliver the capacity and the congestion threshold that triggers the need for capacity delivery. Monte Carlo simulation is used to take into account multiple sources of uncertainty. The model shows that a strategy of capacity delivery based on small increments and short response times can yield more benefits than strategies that consider large capacity increases and long response times. Furthermore, in the specific airport example considered here, it was found that a congestion threshold of $75 \%$ should be the trigger for capacity enlargements if strategies based on small capacity increments and 1 or 5 years to increase capacity are considered. The lesson for decision-makers is that congestion delays must be addressed with foresight.

Bruno Miller is a doctoral student in Air Transportation Systems at the Department of Aeronautics and Astronautics at the Massachusetts Institute of Technology in Cambridge, Massachusetts, United States.

John-Paul Clarke is Assistant Professor in the Department of Aeronautics and Astronautics at the Massachusetts Institute of Technology in Cambridge, Massachusetts, United States.

*Presented at the $7^{\text {th }}$ International Conference on Technology Policy and Innovation in Monterrey, Mexico, June $10^{\text {th }}-13^{\text {th }}, 2003$. Selected for publication in a special volume of the journal Technological Forecasting and Social Change. 


\section{Introduction}

Air transportation is a key strategic asset in that it provides access to markets and thereby enables the economic development of nations and regions [1]. Thus, in order to maintain their competitiveness in a global economy, countries must invest in air transportation infrastructure to ensure their ability to meet current and future demand for aviation services.

Investments in air transportation infrastructure are difficult to evaluate for a variety of reasons, but two are especially worth noting. First, investments in aviation infrastructure must be considered as part of a wider national strategy that involves many other sectors [2]. Second, the size of these investments is typically large and the time for implementation is also usually long. Thus, the benefits of the investment may not be realized for many years [3]. Given these difficulties, investments in air transportation infrastructure are often not implemented or are implemented too late relative to the needs of society.

The methodology presented in this paper is based on the assumption that it is useful to consider investments in aviation infrastructure not as an end in themselves, but rather as a way to provide options to accommodate future growth scenarios. In general, current thinking considers the benefits of infrastructure in terms of the function it was built to perform; however, there are at least three other types of benefits that infrastructures can provide: a) the ability to respond quickly to changes in the market, b) the ability to explore markets, and c) the ability to influence markets. By considering the strategic value of these three benefits, decision-makers can better understand the overall benefits of the infrastructure and justify its delivery.

\section{Objective}

The objective of this paper is to develop and illustrate a methodology for evaluating the strategic value of air transportation infrastructure, i.e. the benefits associated with the ability to react quickly to changes in the market, to explore new markets and to stimulate existing markets. The hypothesis is that by recognizing and taking advantage of this strategic value, it may be possible to design better policies for aviation infrastructure delivery. 
The simulation developed in this study focuses primarily on capturing the benefits due to the ability to react quickly to changes in the market. The simulation can be enhanced to also incorporate the benefits from exploring and stimulating markets. These enhancements have not been elaborated here mostly to simplify the calculations and the presentation of the results; however, at the end of the paper, we indicate how the model could be expanded to incorporate these other benefits.

The next section explains the methodology employed to analyze the strategic value of air transportation infrastructure. Then, numerical results and sensitivity analysis for the specific example considered here are presented. The paper concludes with a summary of the main points and a discussion of the implications of the results for policy-making.

\section{Methodology to calculate the strategic value of aviation infrastructure}

The methodology presented here is based on a combination of system dynamics and Monte Carlo simulation. The first step is to model the infrastructure delivery problem using system dynamics tools. The main advantage of this approach is that it captures the feedback loops and delays that occur in complex systems, such as air transportation. The next step is to determine different strategies for infrastructure delivery and implement them in the system dynamics model. Finally, Monte Carlo simulation is used to take into account multiple sources of uncertainty.

\subsection{Modeling the infrastructure delivery problem with system dynamics}

\subsubsection{Overview of the model}

The example considered in this study is a hypothetical yet common situation in many airports around the world. Here, it is assumed that an element of the air transportation infrastructure (e.g. a runway or a passenger building) is reaching saturation, and, thus, depending on the level of traffic, may lead to congestion (see Figure 1).

[Figure 1 about here] 
In this particular example, Runway capacity $^{l}$ is the limiting factor that leads to congestion. As demand for air travel (Aircraft per hour) increases, the total number of aircraft requesting service on this runway (Total aircraft) also increases. If Runway capacity is held constant, the increase in demand slowly leads to Congestion, which raises the direct operating costs of airlines (Airline congestion cost). The higher operating costs are passed on to the passenger in terms of higher air fares (Air fare impact) and this leads to less demand for travel (Congestion cost loop). In addition, congestion decreases the level of service by lengthening passenger travel time (Level of service impact) which also results in less demand for aviation services (Passenger comfort loop). Once Congestion reaches a certain limit (Congestion threshold), a certain amount of capacity (Capacity increase) is delivered after a certain period of time (Years to increase capacity). These three variables (Congestion threshold, Capacity increase, and Years to increase capacity) are specified by the user and they characterize the different infrastructure delivery strategies (see below). Once capacity is added to the runway, Congestion decreases, thus, stimulating demand by reducing the Air fare impact and Level of service impact. The model assumes that congestion occurs only at a given number of Peak hours per year.

There are two main outputs from this model. The first are the benefits accrued to the airport operator in terms of Airport revenues. Here, it is assumed that they consist mainly of Landing fees paid by the airlines and passenger facility charges $(P F C)$ paid by each traveler. The second output is the cost of infrastructure construction (Delivery cost). Together, these two outputs determine the cash flows of the airport operator and form the basis for calculating the net present value (NPV) for the different strategies (see below).

\subsubsection{Main relationships of the model}

The main relationships used in the model are explained below:

\section{a) Congestion:}

Congestion in this model is defined as the waiting time, Wq, for each user (aircraft) that wants to land on the runway. The waiting time is obtained by modeling this situation as a $\mathrm{M} / \mathrm{G} / 1$ queuing system (i.e. a system with Poisson demand, any type of service time, one server and infinite capacity) [4]:

\footnotetext{
${ }^{1}$ Model variables are written in Italics throughout the whole paper.
} 
$W q=\frac{\lambda \cdot\left(\left(\frac{1}{\mu}\right)^{2}+\sigma_{t}^{2}\right)}{2 \cdot(1-\rho)}$

where $\lambda$ is the average demand rate over a specified period of time determined by the Poisson distribution, $\mu$ is the capacity of the system over the same period of time, $\sigma_{t}$ is the standard deviation of service times and $\rho$ is the "utilization ratio." The metric for congestion used in this study is "hours per peak hour," i.e. hours of waiting time per peak hour of traffic.

The utilization ratio $\rho$ can be defined as the average demand rate over system capacity [5]:

$\rho=\frac{\lambda}{\mu}$

In the model, $\lambda$ is given by Total aircraft and $\mu$ is Runway capacity. A key characteristic of this queuing system is that as $\lambda$ approaches $\mu$, i.e. as demand approaches capacity, the numerator of Equation 1 tends to zero and the waiting time grows non-linearly [5]. For our example, this means that when aircraft demand per hour approaches runway capacity per hour, and, hence the utilization ratio approaches 1, Congestion becomes critical (see Figure 2):

[Figure 2 about here]

b) Annual demand growth rate

Demand for air travel is very volatile and cyclical [6]. In order to capture this behavior, Annual demand was modeled as a mean reverting stochastic process of the form shown in Equation 3:

$d x=\eta \cdot(\mathrm{X}-x) d t+\sigma \cdot d z$ 
where $d x$ is the change in demand $x$ over a time interval $d t, \eta$ is the speed of reversion, i.e. a metric that represents how fast the process returns to its long-term trend, $\mathrm{X}$ is the level to which $\mathrm{x}$ tends to revert, $\sigma$ is the variance parameter and dz represents a Wiener process, i.e. a normally distributed random process (for more details, please consult [7]). This equation was calibrated with historical data for air travel demand in the Unites States between 1979 and 2001 contained in the Form 41 database [8] and using the method outlined in [7]. The long-term annual demand growth rate found was on the order of $3.8 \%$ per year. Two sample paths of this mean reverting process are illustrated in Figure 3:

[Figure 3 about here]

c) Air fare impact and Level of service impact

The Air fare impact and the Level of service impact on air travel demand were determined using the concepts of price and time elasticities, respectively [9]. The price elasticity of demand is defined as the percentage change in total demand that occurs with a $1 \%$ change in average airfare. Similarly, time elasticity of demand is the percentage change in total travel demand that occurs with a $1 \%$ change in travel time. In this study, the Air fare impact is the change in demand from a percentage change in average travel cost times price elasticity (see Equation 4):

Air fare impact $=\varepsilon_{\text {price }} \cdot \Delta$ Cost ${ }_{\text {Travel }}$

where $\varepsilon_{\text {price }}$ is the price elasticity of demand and $\Delta \operatorname{Cost}_{\text {Travel }}$ is the percentage increase in travel cost due to the congestion costs that the airlines are able to pass on to the consumers. Historically, price elasticity of demand has been estimated at approximately -1.6 for leisure passengers and on the order of -0.8 for business passengers [9]. $\Delta \operatorname{Cost}_{\text {Travel }}$ is given by Equation 5:

$\Delta$ Cost $_{\text {Travel }}=\frac{\text { Cost } \text { Congestion }_{\text {Airfare }} / \text { passenger }}{\text { Average }} \cdot$ Cost Transfer 
where $\operatorname{Cost}_{\text {Congestion }} /$ passenger is the average airline operating cost per hour divided by an average number of passengers per flight, Airfare ${ }_{\text {Average }}$ is the average air fare charged per passenger, and $\operatorname{Cost}_{\text {Transfer }}$ is the percentage of Cost $_{\text {Congestion }}$ that the airline is able to pass on to the travelers.

The Level of service impact is the change in demand from a percentage change in average travel time times time elasticity (see Equation 6):

Level of service impact $=\varepsilon_{\text {Time }} \cdot \Delta$ Time $_{\text {Travel }}$

where $\varepsilon_{\text {time }}$ is the time elasticity of demand and $\Delta$ Time $_{\text {Travel }}$ is the percentage change in travel time due to congestion. Time elasticity of demand is nominally considered to be around -0.8 for leisure passengers and approximately -1.6 for business passengers [9].

\section{d) Capacity delivery costs}

In this model, airport capacity is given in terms of aircraft per hour. Capacity delivery costs are obtained by multiplying Capacity increase times a unit capacity delivery cost in US\$/aircraft per hour. Here, the unit capacity delivery cost is assumed to be US\$5 million/aircraft per hour. For example, this means that an increase of 20 aircraft per hour (i.e. 50\% of the assumed runway capacity of the airport in the model) requires an investment of US\$100 million. Runway construction costs vary substantially from airport to airport, ranging anywhere from US\$60 million to US\$300 million, and even more in some particular cases [10]. Thus, the unit capacity delivery cost of US\$5 million/aircraft per hour is just meant to be an approximation to costs in the real world.

e) Congestion threshold, Capacity increase, Years to increase capacity

These constants are the main control parameters to specify the different infrastructure development strategies. Congestion threshold is the maximum level of congestion allowed before the airport operator decides to add more capacity. Capacity increase is the amount of capacity to be added once the decision 
has been taken to deliver more capacity. Finally, Years to increase capacity determine the delay in supplying the desired capacity once the decision has been made to deliver it.

\subsubsection{Model calibration}

The numbers used to calibrate the model are meant to illustrate a realistic situation but they do not represent an actual airport. The airport in this study is assumed to be a one runway facility that serves primarily narrow-body aircraft. The current runway capacity was set at 40 aircraft per hour. Landing fees were estimated at $\$ 200$ per aircraft based on data in [5] and the typical weight of narrow-body aircraft. It is further assumed that congestion occurs only at peak hours and there are 1000 peak hours in a year. The simulation time period is in years and each run covers 50 years.

\subsection{Infrastructure delivery strategies}

For the purposes of this study, each infrastructure delivery strategy is defined by three parameters:

\section{1) Congestion threshold}

This constant determines the maximum level of congestion allowed ("trigger") before the airport operator decides to put more capacity in place. A low threshold reflects a proactive strategy by which the operator intends to have enough capacity to meet demand and, thus, be able to capture more revenues. The disadvantage of such approach is that if demand does not grow as expected, the new capacity may remain unused. A high threshold represents a reactive strategy. Here, an airport operator would wait until it is obvious that the current levels of demand require more capacity. The disadvantages of this strategy are the negative effects of congestion on travel demand explained above.

Each congestion threshold corresponds to a given utilization ratio, $\rho$, as determined by Equation 1 and shown in Figure 2. Five congestion thresholds were considered and are presented in Table 1:

[Table 1 about here] 


\section{2) Capacity increase}

Another strategic decision by the airport operator is how much capacity to add. In order to simplify the analysis, only three cases were considered: $10 \%, 25 \%$ and $50 \%$ increase of existing capacity. The first alternative (10\%) represents a very incremental approach, in which little additions to capacity are made at any given time. For example, modifications to existing approach procedures and/or better sequencing of arriving aircraft enabled by automation support tools may lead to these small increments. The second alternative $(25 \%)$ may include the installation or upgrade of Instrument Landing Systems (ILS) and/or expansion of taxiways and holding areas, for example. The third case (50\%) represents a larger investment like a completely new taxi-way or runway.

The advantage of small capacity increments is that demand may be matched more closely to capacity, thus, avoiding the delivery of unnecessary infrastructure. In addition, capital expenditures are smaller and spread over time, which may be easier to finance and justify than large investments performed at once. Even though it may not be entirely realistic to have a purely incremental strategy throughout the life of an airport, as it is not possible to add incremental capacity by building little pieces of runway at a time, for example, this strategy illustrates a limiting case. The medium and large capacity increments lead to more significant investments at particular points in time. These are more representative of what is seen in practice, as airport capacity enhancements projects tend to be large.

\section{3) Years to increase capacity}

The time that it takes to increase capacity reflects how fast the airport operator can react to changes in the market place. Four cases are considered in this study: 1 year, 5 years, 10 years and 15 years. It is assumed that the time to increase capacity depends on the "level of preparedness" of the operator to respond to changes. A short time to increase capacity means that the airport operator has invested resources a priori to prepare its facilities for expansion. 
If we consider the example of building a new runway, the different Years to increase capacity may correspond to the level of preparedness shown in Table 2:

[Table 2 about here]

The implication with these assumptions is that in order to be able to respond faster, the operator of the facility must have invested some resources beforehand. Two important considerations arise from this observation. First, the level of a priori investment depends on the benefits that reacting quickly brings. Consequently, if the purpose of the a priori investment is to maximize the economic returns of the whole project, the maximum amount that somebody should be willing to invest a priori for the ability to react quickly is the difference between the benefits derived from reacting quickly compared to the benefits under the "business-as-usual" scenario.

The second consideration is that the a priori investment may lower the cost of reacting quickly in the future. For example, buying land for another runway may be less expensive before traffic levels require the addition of this extra runway. If the airport waits until its demand requires another runway, the current owner of the land intended for the extra runway is likely to increase the price of the land because this situation represents a seller's market. Consequently, the premium associated with the a priori investment to obtain the ability to react quickly may be compensated with lower capacity delivery costs.

In order to illustrate this point, two different construction cost schemes were considered. The first one, the so-called "Zero cost difference," assumes no difference in capacity delivery costs. The second one, the so-called "Moderate cost difference," assumes the following reductions in capacity delivery costs per unit of capacity (see Table 3):

[Table 3 about here] 
Notice that there is no difference in delivery cost for 10 years to increase capacity because this is the "business-as-usual" scenario (see below). In addition, it is assumed that having a longer time to deliver capacity (15 years) increases the delivery costs.

\subsubsection{Summary of delivery strategies}

The study considered all possible combinations of the above parameters (Congestion threshold, Capacity increase and Years to increase capacity) for a total of 60 delivery strategies (see Figure 4).

[Figure 4 about here]

Even though it may lead to some unlikely scenarios, such as adding $50 \%$ of capacity every year, the test matrix does span a representative sample of realistic strategies with some limiting cases. Typical strategies seen in the real world arguably fall between $25 \%$ and 50\% Capacity increase with 10 to 15 Years to increase capacity, with a Congestion threshold of $90 \%$ to $95 \%$.

\subsection{Monte Carlo simulation}

Monte Carlo simulation is used to incorporate multiple sources of uncertainty into the calculations. Here, the main uncertainties considered were related to Annual demand growth rate, Air fare impact and Level of service impact. Table 4 shows the variables selected for the Monte Carlo simulation along with their assumed probability distributions and limiting values:

[Table 4 about here]

As explained above, Annual demand growth rate is modeled stochastically. In order to get different random distributions of this variable, the seed parameter is changed between the limits shown in Table 4. Average time refers to the assumed average trip time to the hypothetical airport, which is the basis to calculate the time sensitivity of demand and, hence, the Level of service impact. The time and price elasticities determine the impact of congestion on travel demand because of increased travel time and higher air fares. The 
elasticities are assumed to be between -1.6 and -0.8 [9]. Cost $t_{\text {transfer }}$ reflects the proportion of the increased operating costs due to congestion that the airlines are able to pass on to the passengers. It is assumed that this value oscillates between $60 \%$ and $90 \%$.

A Monte Carlo simulation was run for each of the delivery strategies shown in Figure 4. Each simulation consisted of 7,500 runs. The output of each simulation is a distribution of Airport revenues and Delivery costs, which are then used to calculate the net present value of each delivery strategy (see below).

\subsection{Evaluation of the delivery strategies}

The Monte Carlo simulation generates a distribution of Airport revenues and Delivery costs for each delivery strategy considered. For each run in the simulation, the present value of Airport revenues and Delivery costs is calculated, thus, a distribution of present values of revenues and present values of costs is obtained. The expected net present value for each delivery strategy $i$ is the difference between the mean of the present value of revenues minus the mean of the present value of costs (see Equation 7):

Expected $N P V_{i}=E\left[\right.$ Present Value $\left.(\text { Revenues })_{i}\right]-E\left[\right.$ Present Value $\left.(\text { Delivery Costs })_{i}\right]$

The standard deviation of the net present value for each strategy $i$ is given by Equation 8:

$\sigma_{i}=\sqrt{\sigma_{\mathrm{Re} \text { venues }, i}^{2}+\sigma_{\text {Del.Costs }, i}^{2}+2 \cdot \operatorname{Cov}_{\mathrm{Re} \text { venues }, i ; \text { Del.Costs }, i}}$

where $\sigma_{\text {Revenues,i }}$ and $\sigma_{\text {Del.Costs,i }}$ are the standard deviations of the present values of Airport revenues and Delivery costs for strategy i, respectively, and $\operatorname{Cov}_{\text {Revenues,i; Del.Cost,i }}$ is the covariance between the present value of Airport revenues and Delivery costs for strategy i. 
The calculation of the present values requires the selection of a discount rate. For the purposes of this study, a discount rate of $10 \%$ was chosen. There is always some uncertainty about what the discount rate should be, therefore, sensitivity analyses were also performed (see below).

In order to determine the strategic value of being able to react quickly, the expected NPV for each delivery strategy was compared to a baseline case that exemplifies a "business-as-usual" situation. The baseline case was assumed to be the strategy were $50 \%$ of the current capacity is delivered every 10years after a congestion threshold of $90 \%$ has been reached. Consequently, the expected value of quick reaction for strategy $i$ is given by Equation 9:

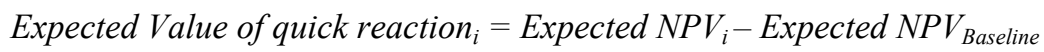

Remember that the expected value of quick reaction is also the maximum amount of a priori investment that an airport operator is willing to spend to implement strategy i. The standard deviation of the expected value for strategy $\mathrm{i}$ is given by Equation 10:

$\sigma_{\text {Expected Value of quick reaction }}=\sqrt{\sigma_{i}^{2}+\sigma_{\text {Baseline }}^{2}}$

where $\sigma_{\mathrm{i}}$ and $\sigma_{\text {Baseline }}$ are the standard deviations of the expected NPV of strategy $i$ and the expected NPV of the baseline scenario, respectively.

For delivery strategies that consider 15 years to increase capacity, Equations 9 and 10 would compute the "expected value of delayed reaction" and its standard deviation, respectively, as opposed to the value to react quickly. These cases are considered here because there may be advantages to delaying investments. 


\section{Results}

\subsection{Zero cost difference}

The results for the situations where there are no cost difference for delivering capacity quicker are shown below. For the case of 1 year to deliver capacity, the results suggest that strategies involving small increments $(10 \%$ capacity increase) lead to higher expected values to react quickly than strategies with larger capacity increments (see Figure 5). For example, for a Congestion threshold of 90\%, the ability to react quickly provides benefits on the order of $\$ 14.2$ million $+/-14$ million. Thus, the airport operator should be willing to spend up to this amount a priori to have the ability to react quickly. Large increases in capacity ( $25 \%$ or $50 \%$ capacity increase) generate, on average, negative expected values for the ability to react quickly. The intuition behind this is that larger expansions may result in over-capacity that is not utilized until some years after it has been delivered and, thus, the investment is more difficult to recover.

\section{[Figure 5 about here]}

The results further show a large standard deviation and a strong dependence on the Congestion threshold. The large standard deviation can be explained by the fact that since capacity is being delivered very quickly, the system is better able to accommodate increases in demand. This is a reinforcing loop in which the system delivers more capacity as demand grows, thereby stimulating even more traffic. When the time to deliver capacity is longer, the system can not react as quickly to changes in demand and, therefore, the variability of traffic is reduced (see below). The dependence of the results on the Congestion threshold arises from the way that present values are determined. Higher Congestion threshold mean that capital expenditures come further in the future and, thus, they get discounted more. The extra revenues from an earlier capacity expansion are not large enough to offset the higher present cost of the earlier investment. Therefore, with higher Congestion threshold and 1 year to increase capacity, the expected value of the ability to react quickly increases. 
As the time to deliver capacity increases from 1 to 5 years, small capacity increments still lead to higher expected values than larger capacity increments (see Figure 6). Notice, however, that the standard deviation and the dependence on the Congestion threshold are less than in the case of 1 year to increase capacity. Because the response time to deliver capacity is longer, demand growth has is limited and, therefore, it does not vary as much as when capacity can be delivered within a year. Furthermore, the longer time to add capacity allows demand to grow to levels that make more use of the new capacity. Therefore, on average, the expected value to react quickly is larger for strategies that have 5 years to increase capacity compared to strategies with 1 year to increase capacity.

[Figure 6 about here]

For longer times to deliver capacity (15 years), similar observations as in the previous cases can be made. Smaller capacity increases lead to higher expected values for the ability to delay reaction than larger capacity increases (see Figure 7). Furthermore, the variability of the results and the dependence on the Congestion threshold are also less. Notice that the expected value of delayed reaction is positive in almost all cases. Because it takes so long to deliver the new capacity, demand is very likely to grow to levels where the added capacity will be completely utilized in almost all cases, thus, recovering the investment very quickly.

[Figure 7 about here]

In summary, for a capacity delivery scheme in which there is no cost reductions associated with delivering capacity quickly, the strategy that results in the highest value for the ability to react quickly is to increase capacity in small increments (10\% capacity increase) with 5 years to deliver capacity, regardless of the congestion threshold. Lower time to increase capacity, i.e. 1 year, may lead to positive values for the ability to react quickly only if the congestion threshold is high. For strategies with 15 years to deliver capacity, the expected value of delayed reaction is similar to the expected value of the ability to react quickly with 5 years to deliver capacity, independent of the congestion threshold. 


\subsection{Moderate cost difference}

The results for the cases where there is a moderate difference in the delivery costs per unit of capacity for strategies with lower time to increase capacity are shown below. For example, for strategies with 1 year to deliver capacity, the ability to react quickly becomes very valuable in all cases (see Figure 8). Similarly to what was shown in the previous sub-section, the expected value of the ability to react quickly increases and has lower variability with smaller capacity increases.

[Figure 8 about here]

Notice that the expected value of quick reaction depends significantly on the Congestion threshold. In fact, there seems to be an optimal Congestion threshold of approximately $75 \%$ utilization ratio for all of the capacity increases with 1 year to deliver capacity. This is explained by the observation that Congestion thresholds lower than $75 \%$ probably lead to more capacity delivered than is needed to meet demand, thus resulting in expensive over-capacity. Moreover, higher Congestion thresholds imply that the negative effects of increased congestion are larger than the savings in present delivery costs accrued by delaying the investment. Thus, if it takes 1 year to deliver capacity, the optimal strategy for an airport operator would be to increase capacity in small to medium increments $(10 \%$ or $25 \%)$ with a Congestion threshold of $75 \%$.

Similar observations can be made when there are 5 years to deliver capacity (see Figure 9). Here, the best strategies would also be to deliver small or medium increments of capacity with a Congestion threshold of $75 \%$. Notice, however, that the expected values to react quickly in these cases are much lower than in the case when there is 1 year to deliver capacity. This is probably a reflection of the assumption that the delivery cost per unit of capacity is less for strategies with 1 year to increase capacity; however, remember that in order to gain the ability to react within 1 year, the airport operator would also have to make a larger a priori investment. Thus, the net benefits of both strategies may be comparable.

[Figure 9 abut here] 
For strategies with 15 years to deliver capacity, the value of delayed reaction is positive for small and medium increments in capacity (see Figure 10). Contrary to the two cases discussed above, there is not an optimal Congestion threshold here. The only observation that can be made is that a higher Congestion threshold seems to yield higher values of delayed reaction. Notice that, overall, the expected value of delayed reaction is much lower than the expected value to react quickly with 1 or 5 years to deliver capacity.

[Figure 10 about here]

In summary, if there are moderate cost reductions associated with faster capacity delivery, the optimal policies would be to deliver small to medium capacity increases with a congestion threshold of approximately $75 \%$. The strategies with 1 year to deliver capacity generate the most expected value of quick reaction, but the net benefits may be comparable to strategies with 5 years to increase capacity because the a priori investment for being able to react within 1 year should be larger.

\section{Sensitivity analysis}

The main reason to perform sensitivity analysis in this study is to determine the effect of changes in the discount rate on the expected values of the ability to react quickly or delay reaction. The other principal sources of uncertainty are taken into account in the Monte Carlo simulation and require no further testing. The sensitivity analysis measures the percentage change on the expected value to react quickly or delay reaction due to a $1 \%$ change in the discount rate. A sensitivity on the order of 1 means that the results are very sensitive and a sensitivity of zero denotes no impact of the discount rate on the results. A negative sensitivity implies that the change is in the opposite direction as the change in the discount rate.

The sensitivity analysis was performed by considering the effect of a $-20 \%$ and $+20 \%$ change in the discount rate, i.e. changes due to a discount rate of $8 \%$ and of $12 \%$, respectively (recall that the baseline discount rate was $10 \%$ ). The analysis was performed on all strategies with 5 years to deliver capacity. 
Figure 11 shows the sensitivity to a discount rate of $8 \%$. In general terms, the sensitivity of the results is very high and this sensitivity increases with larger capacity increments and with decreasing congestion thresholds. For small capacity increments, the sensitivity is on the order of -0.9 for low congestion thresholds and 0.1 for a congestion threshold of $95 \%$. Large capacity increments show high sensitivity, especially the case for a congestion threshold of $60 \%$ which has a sensitivity on the order of -35 .

[Figure 11 about here]

It is not surprising that the larger capacity increments are more sensitive to the discount rate because they imply the largest capital expenditures and, thus, their absolute change in magnitude is larger. Lower congestion thresholds mean that there may be more investments, all else held equal, and, therefore, the absolute change in the magnitude of the investments will also be larger.

The sensitivity to a discount rate of $12 \%$ is shown in Figure 12. The same observations as for the case of an $8 \%$ discount rate hold: sensitivity is very high and it increases with larger capacity increments and lower congestion thresholds.

[Figure 12 about here]

The sensitivity analysis clearly shows that the choice of discount rate is of critical importance. This is an aspect that deserves further investigation; however, rather than a weakness of the system dynamics and Monte Carlo simulation methodology presented here, the high sensitivity to the discount rate is a reflection of the great care that must be taken when using discounted cash flow and net present value to evaluate investments. Furthermore, this analysis shows that larger investments are more sensitive to the discount rate than smaller investments, which may be a further argument towards smaller-scale capacity enhancements. 


\section{Conclusions}

The model results suggest that the strategic value of air transportation infrastructure can be significant. By adopting strategies that take advantage of the ability to react quickly to changes in the market, the value of a piece of infrastructure can be increased. In this study, it was shown that a strategy of capacity delivery based on small increments and short response times can yield more benefits than strategies that consider large capacity increases and long response times; however, the ability to react quickly implies an a priori investment. The difference between the expected value from the strategy with the ability to react quickly and the "business as usual" strategy is the maximum that a rational investor should be willing to invest a priori. The strategic value of reacting quickly increases if there is a moderate cost reduction in the delivery costs as compared to strategies with long time to increase capacity.

A critical decision in any infrastructure capacity investments is when to begin work to add the capacity. This study shows that there may be an optimal congestion threshold to begin adding capacity depending on what strategy is being followed. In the specific airport example considered here, it was found that a Congestion threshold of $75 \%$ should be the trigger for capacity enlargements if strategies based on small capacity increments and 1 or 5 years to increase capacity are considered. The lesson for decision-makers is that congestion delays must be addressed with foresight. It may be too late to do something once congestion becomes critical. Thus, depending on the expectations for future growth in air travel, the value of being able to react quickly to changes in the market may be substantial.

The methodology described here can be expanded to evaluate the other two components of the strategic value of air transportation infrastructure identified above, i.e. the ability to create and to stimulate markets. The key is to introduce these processes into the system dynamics model, because once this is accomplished, the Monte Carlo simulation and the evaluation of strategies as described above can be used. The ability to create markets can be investigated by simulating the demand for air travel within a competitive market that allows airlines to enter and leave the market served by the airport in the system dynamics model. The entry/exit of airlines would depend, among other factors, on the congestion levels at the airport and, thus, on the infrastructure investments. Moreover, the ability to stimulate markets can be analyzed by allowing 
airlines in the competitive market model to expand and contract flight schedules. Again, congestion levels at the airport would be a key element in the airline's decision to alter its operations.

\section{References}

[1] ICAO: Impact of Civil Aviation on State's Economies. World-wide CNS/ATM Systems Implementation Conference, Rio de Janeiro, 1998.

[2] Kessides, Christine: A review of Infrastructure's Impact on Economic Development, in Infrastructure and the Complexity of Economic Development, Berlin: Springer Verlag, 1996.

[3] Kapur, Anil: Airport Infrastructure: The Emerging Role of the Private Sector. World Bank Technical Paper No. 313. Washington, D. C., 1995.

[4] Larson, Richard and Amedeo R. Odoni: Urban Operations Research. New Jersey: Prentice-Hall, 1981.

[5] de Neufville, Richard and Amedeo Odoni: Airport Systems - Planning, Design and Management. McGraw-Hill, 2003.

[6] Skinner, Steve, Alex Dichter, Paul Langley and Hendrik Sabert: Managing Growth and Profitability across Peaks and Troughs of the Airline Industry Cycle - An Industry Dynamics Approach, in Handbook of Airline Finance, Gail F. Butler and Martin R. Keller, eds., MacGraw-Hill, 1999.

[7] Dixit, Avinash K, and Robert S. Pindyck: Investment under uncertainty. Princeton, New Jersey: Princeton University Press, 1994.

[8] USDOT: Form 41, US Department of Transportation, Bureau of Transportation Statistics, Office of Airline Information, 1979-2001. 
[9] Belobaba, Peter: Characteristics of Air Transportation Markets and Demand for Air Travel. Lecture notes from The Airline Industry course taught at the Massachusetts Institute of Technology, 2001.

[10] FAA: Aviation Capacity Enhancement Plan. Federal Aviation Administration, Office of System Capacity, Washington, D. C., 1992-2001. Available at: http://wwwl.faa.gov/ats/asc/ACE.html 
Tables:

Table 1: Relationship between congestion threshold and utilization ratio for the example analyzed in this study.

\begin{tabular}{|c|c|}
\hline Congestion threshold (hr/peak hr) & Utilization ratio, $\rho$ \\
\hline
\end{tabular}

\begin{tabular}{|c|c|}
\hline 0.019 & $60 \%$ \\
\hline 0.029 & $70 \%$ \\
\hline 0.038 & $75 \%$ \\
\hline 0.113 & $90 \%$ \\
\hline 0.239 & $95 \%$ \\
\hline
\end{tabular}


Table 2: Hypothetical relationship between Years to increase capacity and level of preparedness for the process of building a runway.

\begin{tabular}{|c|c|c|}
\hline \multirow{2}{*}{$\begin{array}{c}\text { Years to increase } \\
\text { capacity }\end{array}$} & \multicolumn{2}{|c|}{ Level of preparedness } \\
\hline & A priori investments & Outstanding tasks \\
\hline 1 & $\begin{array}{ll}- & \text { Purchase land } \\
\text { - } & \text { Permitting process completed } \\
\text { - } & \text { Runway is built }\end{array}$ & - $\quad$ Install navigation aids \\
\hline 5 & $\begin{array}{ll}- & \text { Own land } \\
- & \text { Permitting process completed }\end{array}$ & $\begin{array}{ll}- & \text { Build runway } \\
- & \text { Install navigation aids } \\
\end{array}$ \\
\hline 10 & - $\quad$ Purchase land & $\begin{array}{ll}- & \text { Permitting process } \\
- & \text { Build runway } \\
- & \text { Install navigation aids } \\
\end{array}$ \\
\hline 15 & & $\begin{array}{ll}- & \text { Purchase land } \\
- & \text { Permitting process } \\
- & \text { Build runway } \\
- & \text { Install navigation aids }\end{array}$ \\
\hline
\end{tabular}


Table 3: Differences in capacity delivery cost for the Moderate cost difference scheme. Years to increase capacity Reduction in capacity delivery cost/unit of capacity

\begin{tabular}{|c|c|}
\hline 1 & $-50 \%$ \\
\hline 5 & $-25 \%$ \\
\hline 10 & $0 \%$ \\
\hline 15 & $+25 \%$ \\
\hline
\end{tabular}


Table 4: Variables considered for the Monte Carlo simulation and their assumed probability distributions.

\begin{tabular}{|l|c|c|c|c|}
\hline \multicolumn{1}{|c|}{ Variable } & Units & Probability Distribution & Max. value & Min. value \\
\hline Annual demand growth rate seed & N/A & Uniform & 1 & 10,000 \\
\hline Average travel time & Hours & Uniform & 2 & 4 \\
\hline Time elasticity & N/A & Uniform & -1.6 & -0.8 \\
\hline Price elasticity & N/A & Uniform & -1.6 & -0.8 \\
\hline Cost $_{\text {transfer }}$ & $\%$ & Uniform & $60 \%$ & $90 \%$ \\
\hline
\end{tabular}




\section{Figures:}

Figure 1: System dynamics model of the hypothetical situation considered in this study.

Figure 2: Waiting time (in terms of "hours of waiting" per hour) of an $\mathrm{M} / \mathrm{G} / 1$ system as a function of utilization ratio for the parameters $(\lambda, \mu$ and $\sigma)$ chosen in this model.

Figure 3: Sample paths for air travel demand growth rate as modeled in this study.

Figure 4: Matrix of the delivery strategies analyzed in the study.

Figure 5: Results showing the expected value of quick reaction for strategies with Years to increase capacity equal to 1 as a function of increases in capacity and utilization ratio (Congestion threshold) with the assumption that there is no difference in the delivery costs.

Figure 6: Results showing the expected value of quick reaction for strategies with Years to increase capacity equal to 5 as a function of increases in capacity and utilization ratio (Congestion threshold) with the assumption that there is no difference in the delivery costs.

Figure 7: Results showing the expected value of delayed reaction for strategies with Years to increase capacity equal to 15 as a function of increases in capacity and utilization ratio (Congestion threshold) with the assumption that there is no difference in the delivery costs.

Figure 8: Results showing the expected value of quick reaction for strategies with Years to deliver capacity equal to 1 as a function of increases in capacity and utilization ratio (Congestion threshold) with the assumption that there is moderate difference in the delivery costs. 
Figure 9: Results showing the expected value of quick reaction for strategies with Years to deliver capacity equal to 5 as a function of increases in capacity and utilization ratio (Congestion threshold) with the assumption that there is moderate difference in the delivery costs.

Figure 10: Results showing the expected value of delayed reaction for strategies with Years to deliver capacity equal to 15 as a function of increases in capacity and utilization ratio (Congestion threshold) with the assumption that there is moderate difference in the delivery costs.

Figure 11: Effect of a discount rate of $8 \%$ on the value to react quickly for all strategies considering 5 years to deliver capacity.

Figure 12: Effect of a discount rate of $12 \%$ on the value to react quickly for all strategies considering 5 years to deliver capacity. 
Figure 1

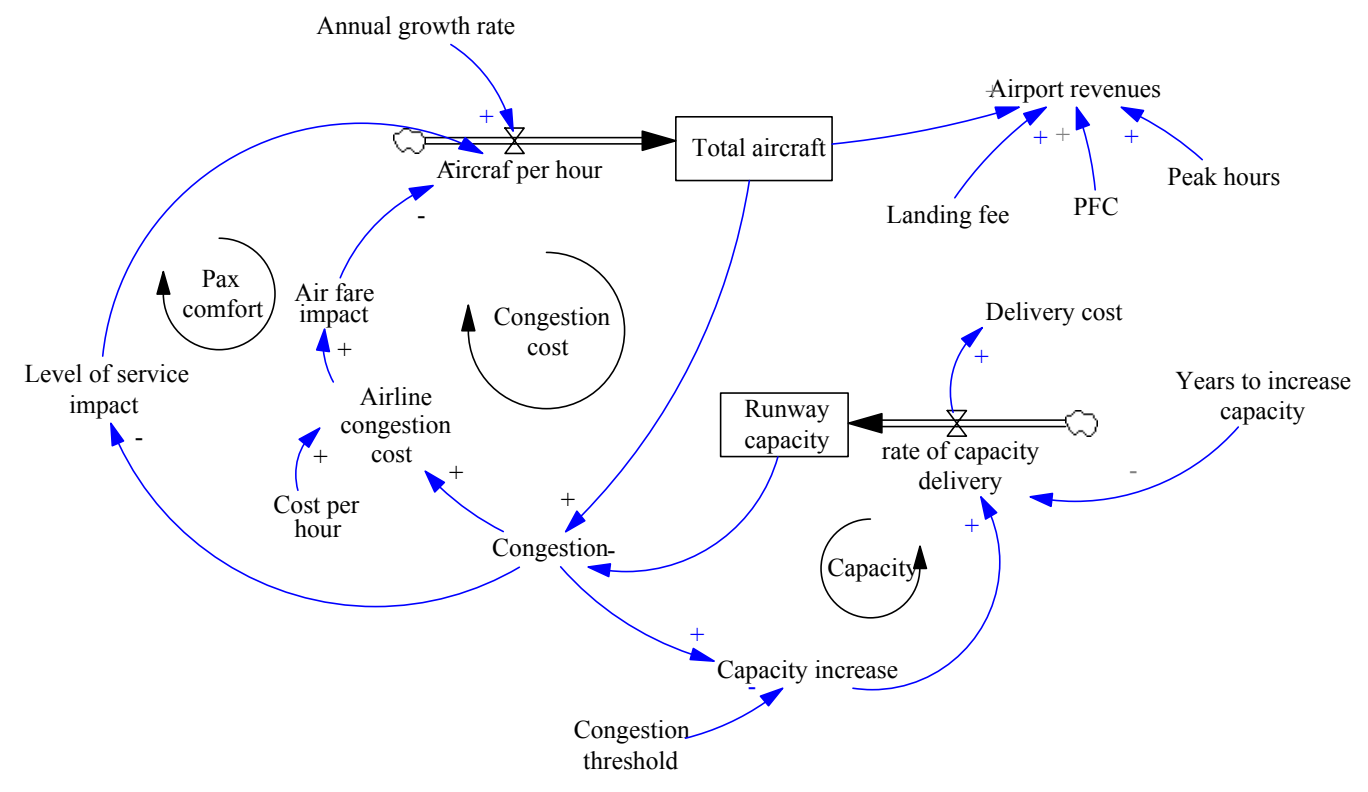


Figure 2

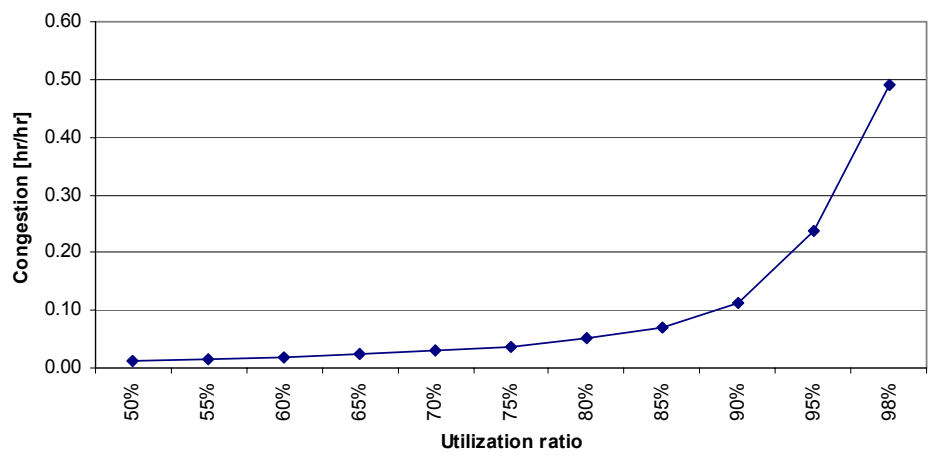


Figure 3

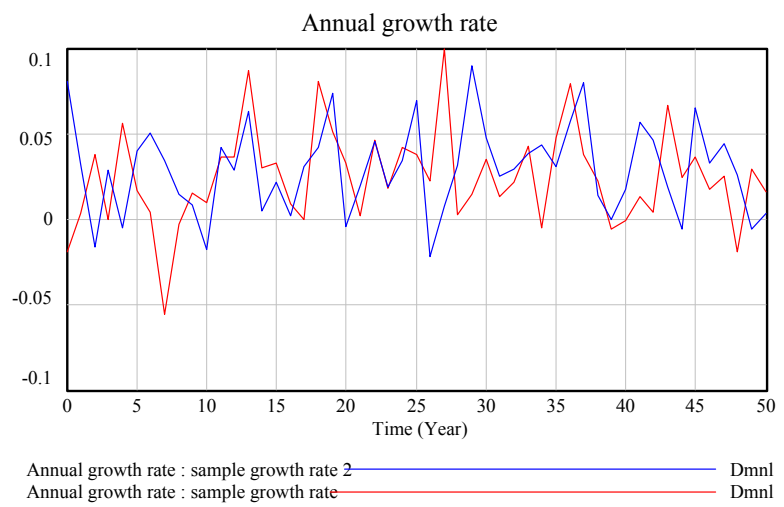


Figure 4

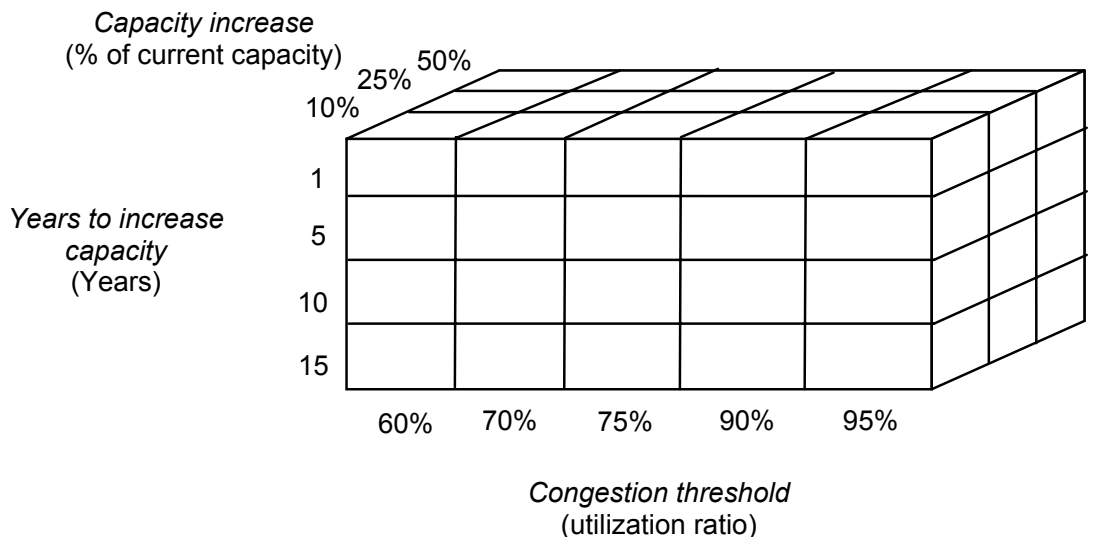


Figure 5

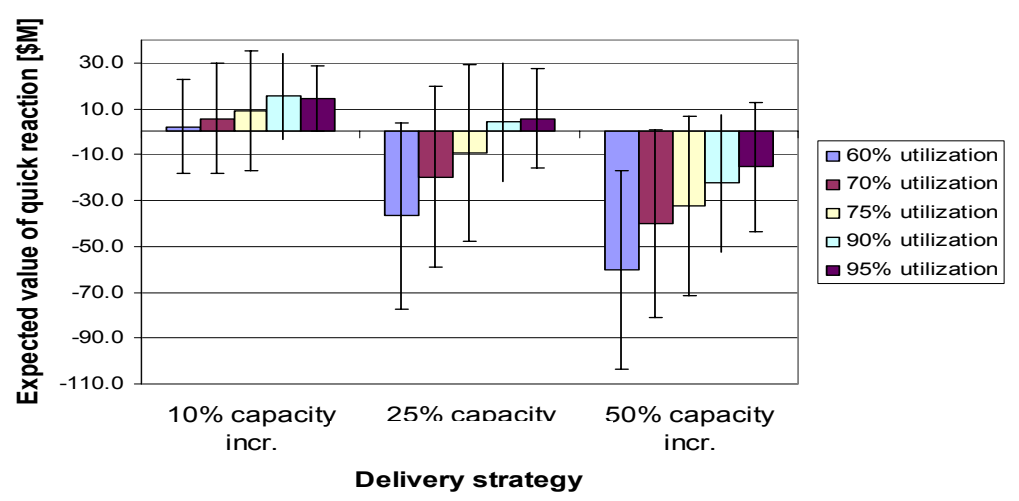


Figure 6

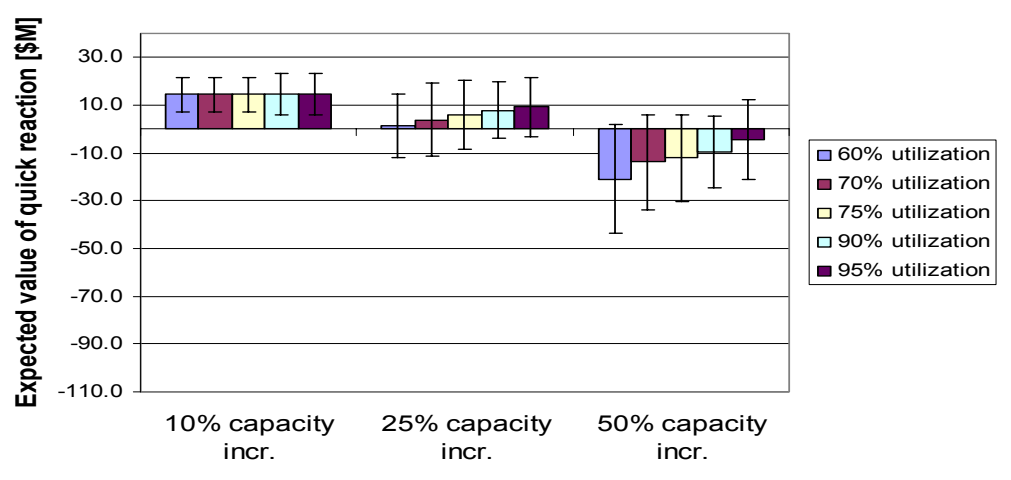


Figure 7

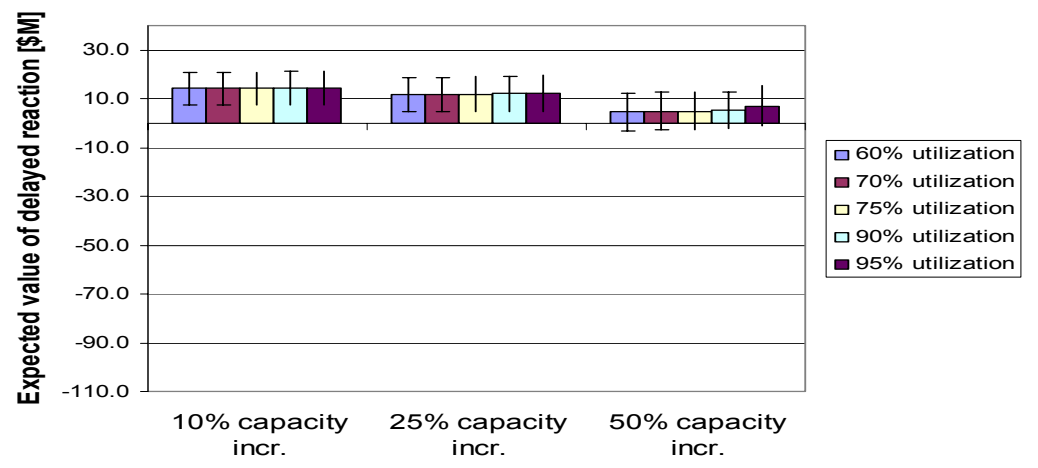


Figure 8

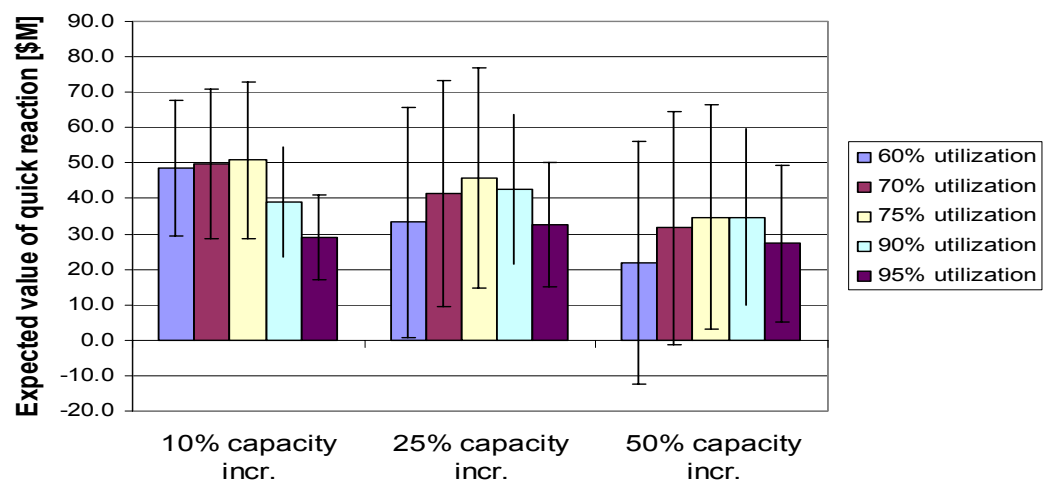


Figure 9

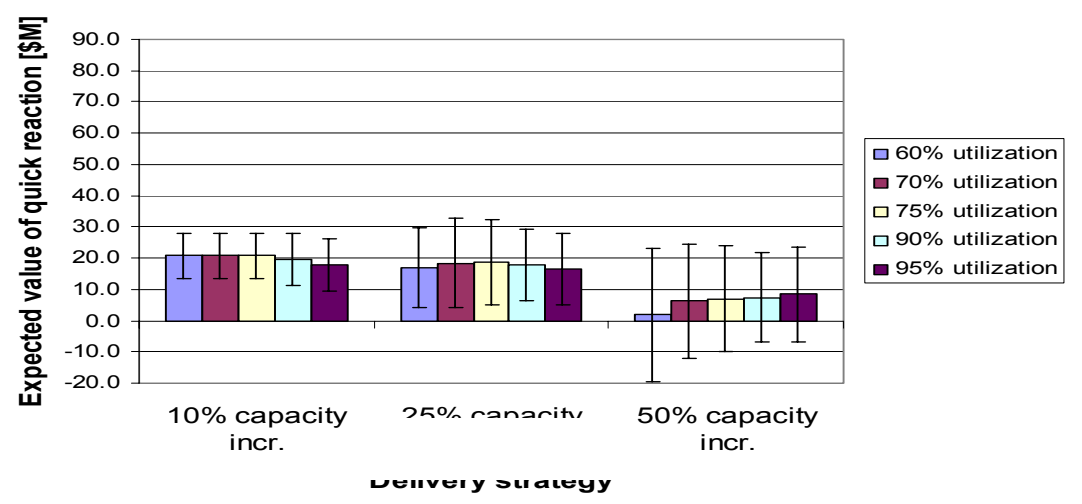


Figure 10

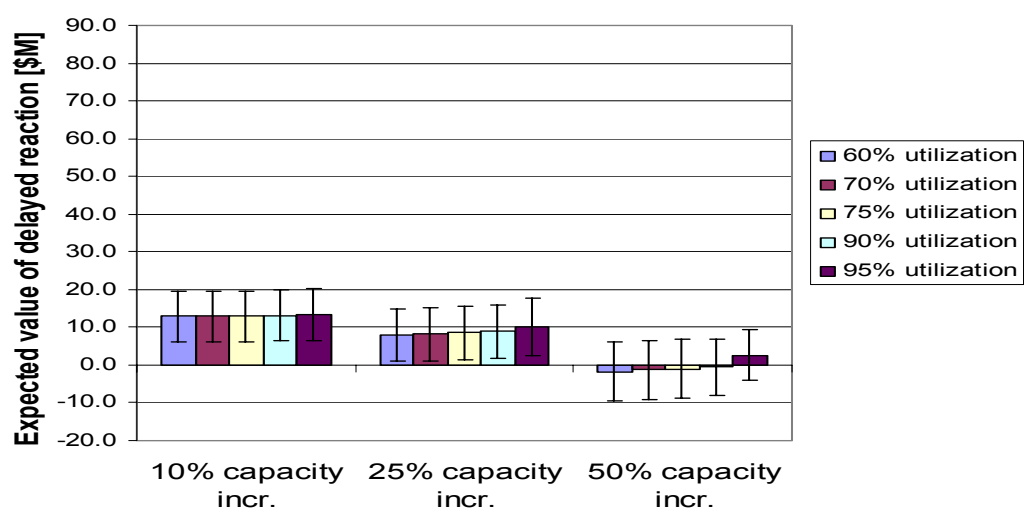


Figure 11

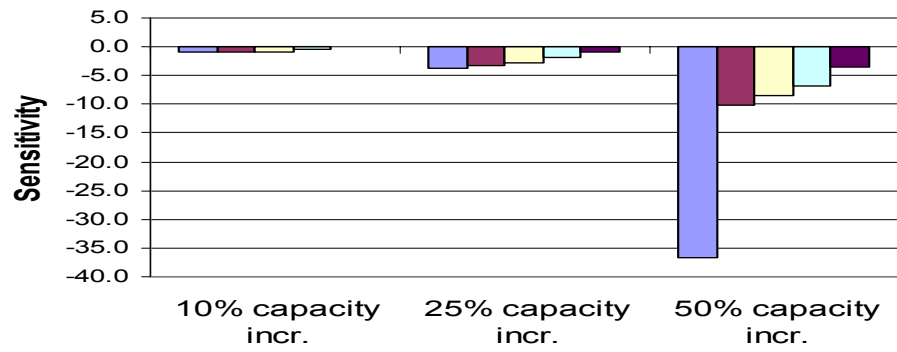

$\square 60 \%$ utilization

- $70 \%$ utilization

$\square 75 \%$ utilization

$\square 90 \%$ utilization

- $95 \%$ utilization

incr.

incr. 
Figure 12

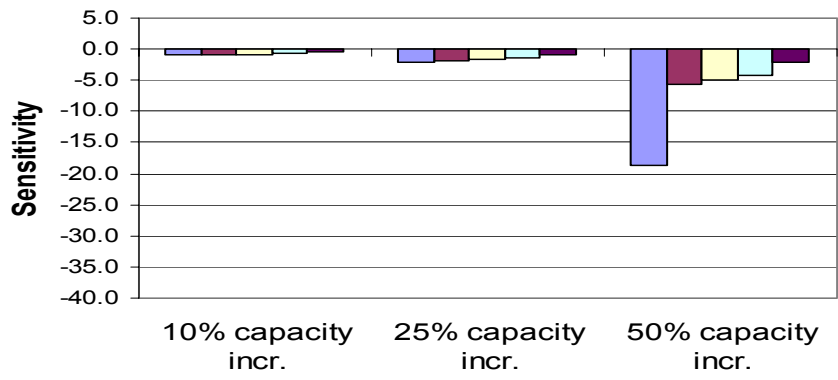

$\square 60 \%$ utilization

$\square 70 \%$ utilization

$\square 75 \%$ utilization

口 $90 \%$ utilization

- $95 \%$ utilization

incr.

incr.

incr. 